\title{
COUNSELLING STRATEGIES FOR LEARNING DEFICIT CHILD IN BORNO STATE, NIGERIA
}

\section{IBRAHIM T. BOKKO, $L P C N, C C N$}

\begin{abstract}
This paper examined some counselling techniques that could be used in counselling and helping students that exhibit learning deficit. Some causes of learning deficit which include environment, chemical, viruses and vitamin deficiencies and types of learning deficit were highlighted as short attention span, preservation, hyperactivity, speech disability, reading disability (dyslexia), to mention but a few. Reinforcement and its schedules were also enumerated as the motivator that can effect change in the client. Shaping, padding, chaining and extinction were suggested as counselling techniques that could be used in handling some students with some categories of learning deficits. It was concluded that counsellors should follow strictly the principles of the counselling technique chosen so that the technique will be effective on the client.
\end{abstract}

\section{Introduction}

Learning Deficit is sometimes referred to as learning disability or learning disorder. For a person to be diagnosed as learning disabled person it means the person has significant learning problems in a given academic area, (Learning Disability, n.d.). However, such disabilities are not enough to diagnose a person officially as having learning disorder. Learning disorder on the other hand are those unknown factors that hinders and or affects the brains ability to receive and process information. Learning disorder can be caused by (Pressinger,1997), environmental and chemical exposure which damages the delicate brain growth process an unborn child during pregnancy or after birth. These exposures can cause hearing disability, attention disorder, hyperactivity, and other child behaviour anomalies (learning disorder). Pressinger, (1997), observed that human brain starts developing at $4^{\text {th }}$ week of pregnancy at the rate of 4,000 cells per second so when chemicals or virus affect fetus, it will disturb the normal development of the brain in its normal position. This declaration puts a question mark on children of the smoking and drinking mothers and those pregnant mothers working in the poisonous environment and the hazardous areas. Ambron, and Salk, (1987), observed that, chemicals can cause a wide range of congenital abnormalities that count for about ten percent of birth deficit. The severity of abnormality depends on the amount of chemical exposure the mother had during pregnancy.

Learning is observed in terms of activity, action, performance, response, and reactions which explain overt behavior of individual. When the individual's overt or observable behaviour manifests deficiencies like mental retardation, childhood autism, childhood schizophrenia, learning disabilities and or other emotional disturbances, one is said to exhibiting learning disability or disorder. These kinds of behaviours are related to the behaviours of other people of the same age. Where people are classified as having behaviour problems and categorized as having behaviour deficiencies, behaviour excesses, and inappropriate behaviours. It is because of such problems and those not mentioned here, experts proposed techniques called, 'behaviour modification'.

Causes of behavior problems are numerous which include; Environmental, Chemical, Viruses and Vitamin deficiencies, (Pressinger, 1997). Martin and Pear, (n.d.), listed some 
common learning disabilities that are found among children. These include; short attention span, preservation, hyperactivity, speech disability, reading disability (dyslexia), autism, antisocial behaviours and toilet training, p. 5 .

\section{Shert attention Span}

Attention span is the amount of time that a person can concentrate on a given task without becoming distracted. The length of human attention span varies depending on age and the task at hand. This explains reasons for allocating different time for course/lesson duration for nursery, primary, secondary and tertiary schools. A nursery class has 20-25 minutes, Primary class has 30-35 minutes, Secondary class has 35-40 minutes and Tertiary lecture has an hour or more to buttress the level of attention span of such categories of learners. One of the causes of lack of attention span is the delayed development of the brain. It is characterized by an impairment to learn, to listen, to wait for one's turn, and to complete a task, (Shama, 2009). Short Attention Span (attention deficit) is lack of ability to put the amount of time expected of a person to concentrate on a task without becoming distracted. It is one of the causes of hyperactivity in children. To improve on the attention span of the child it is recommended that children should be advised to reduce watching Television, Video and Computer games, playing and working on computer to the maximum of two hours a week, (Shama, 2009). This is because such activities rewires the development of brain of the child and increases attention deficit. Instead, children should be taken to parks and to increase their outdoor games which encourages the development of eye-hand coordination, encourage the child to read and have a good night sleep up to 8-9 hours to have enough rest to increase the child's concentration, Shama, (2009).

\section{Preservation}

According to (Hornby) Oxford Advanced Learner's Dictionary, (2010), preservation is the art of keeping something in its original state or in good condition. Martin and Pear, (2006) explained that, a preservation child stares at an item for a lengthy period or stays in a place without moving out for long time. This is a reserved child that cannot be easily socialized. He does not mingle with his classmates; he does not participate in games alongside other students. This kind of student does not explore his environment and has little or no motivation towards learning. It is now the role of the counsellor and of course the teacher to motivate the child to develop the habit of being motivated to explore his immediate environment.

\section{Hyperactivity}

Hyperactivity as described by Wikipedia is a physical state in which a person is abnormally and easily excitable. This is a kind of a child that do not stay at a place, he has too much of activity. This child is labeled as having "Attention Deficit Hyperactivity Disorder" (ADHD) Board of Trustees, 2007). The child is not bad, lazy or stupid academically. Their problems are lack of paying attention and sitting in a place for amount of time to do a given task. They do things haphazardly, have many friends, talk too much, and move round the school compound aimlessly. Such children are destructive and do things in disorganized manner. Morgan, King, Weisz, \& Schopler, (2006), maintained that youngsters with this problem are impulsive and overactive. 


\section{Dyslexia}

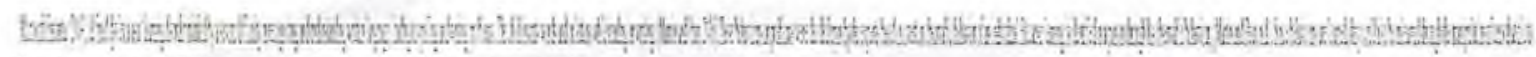

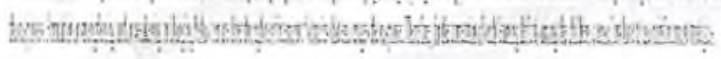

\section{Reinforcement}

Ramalingam, (2006), defined reinforcement as 'a reward that strengthens a response...unconditioned irritant causing biologically meaningful reactions...'Hartney, (2009), opined that, reinforcement is a psychological term that refers to the way behaviours increases or decreases according to whether people associates it with a positive experience (reward) or a negative experience (punishment). While Wikipedia the free encyclopedia (2012), defines reinforcement as a term in operant conditioning and behavior analysis for a process of strengthening a directly measurable dimension of behavior, like, the frequency of behavoiur being exhibited (rate), length of time the behavior being exhibited (duration), interest or motivation of respondent towards the behavior being exhibited (magnitude), and the ability to respond to the stimulus presented (latency). Uwe, (2005), observed that counsellor must be sure that the reinforcement he or she is using is strong enough to motivate the counsellee to perform the desired behaviour.

\section{Types of Reinforcement}

There are two types of reinforcement, Positive Reinforcement and Negative Reinforcement.

\section{Positive Reinforcement}

Ramalingam, (2006), defines positive reinforcement as something provided after a response in order to increase the probability of that response reoccurring in the future. Fritsch. (2009) defined positive reinforcement in a behavioural field of Psychology, that, reinforcement occurs when a reward, sometimes called reinforcement is given for a specific desired behaviour so that there will be increase in the desired behaviour. A positive reinforcement is stimulus that is presented after an operant behaviour for a particular to increase in its frequency. Therefore, positive reinforcement is a technique used to increase desired behaviour.

\section{Negative Reinforcement}

Morgan, et al, (2006), defines negative reinforcement as a stimulus or event which, when its cessation or termination is contingent on a response, increases the likelihood that the response will occur again. Negative reinforcement is served to encourage a certain positive behavior by taking away a negative stimulus. It occurs when something already present is removed (taken away) as a result of which a person behaviours increase because it creates a favorable outcome in that person. Furthermore, when a person's behaviour leads to removal of something that was unpleasant, that removal is negative reinforcement. 


\section{Schedule of Reinforcement}

Morgan, et al, (2006), observe that, the practice of contingent positive reinforcement for every occurrence of a particular behavior is called, 'continuous reinforcement', after when the behavoiur has been learned, it can then be maintained by schedule of reinforcement. Scheduling of reinforcement is intended to increase resistance of such learned bchavior towards extinction. For instance, certification after every successful completion of educational institution.

\section{Types of reinforcement}

Positive Reinforcement: adding of an appetitive stimulus to increase a certain response Positive Punishment: adding of an aversive stimulus to decrease certain response Negative Reinforcement: taking away of an aversive stimulus to increase response Negative Punishment: taking away of an appetitive stimulus to decrease a certain response

\section{Appetitive (pleasant) Aversive (unpleasant) Stimulus Stimulus 0 \\ Present Positive Reinforcement Positive Punishment \\ Take away Negative Punishment Negative Reinforcement}

Obtained from: Boerce (1998: 13).

Considering these schedule of reinforcement, experts programed methods of presentations. These include; ratio schedules, continuous schedules, fixed schedules, variable schedules and several other complex schedules of reinforcement, with its characteristic patterns of responding are used for specific reasons.

\section{Counselling Strategies for the Learning Deficit Child Shaping}

Shaping is the development of a new behavior that is not occurring in the person by successive reinforcement of closer approximation, (Morgan, et al, 2006) and the extinguishing of proceeding approximation of the behavior. It is a procedure that is expected to establish a new behavior that was not formed. Shaping starts with reinforcement of a response that occurs. Onwuasoanya, (2006), maintained that, shaping is an operant technique by which a desired behavior is evoked by reinforcing successive response approximation of that behavior. This is a procedure where by a counsellor detemines level at which the client is about to exhibit the desired behavior termed 'Terminal behaviour'. The counsellor begins by instructing or teaching the client the expected response and encourages the repetition of the behavior in part and continuousto reward it until when it becomes part of the client's behavior. Shaping teaches and reinforces gradual learning, step-by-step learning procedure while extinction of irrelevant responses.

Shaping uses reinforcement like, praises, edible, privileges and social reinforcements, (Martin and Pear, n.d.). It can be used to treat hyperactivity in children when trying to teach or influence a child to seat calm in his seat during the lessons. It can also be used in treating a truant or late coming to school student and the child that has writing and 
drawing problems. Garry and Pear, (n.d.), maintained that, a counsellor that uses shaping as a strategy should not be too fast in moving to the next step of activity, so that the new task or approximation will not extinguish the previously learned approximation.

\section{Fading}

This is a gradual replacement of a stimulus that elicits response in a way that the response will occur to another stimulus. This technique is for the development of appropriate behavior in a client. It can be used in treating autistic and severely retarded children. An Autistic child that mimics a word, to Martin and Pear, (n.d.), could be taught with padding strategy. For instances, to teach a child verbal skills like to pronounce 'cat'. The counsellor can divide the word into 'caa' and 'tuh'. The child will have to imitate 'caa' then you reinforce. When the child mimics 'tuh' you also reinforce. The counsellor should fade out 'tuh' fist before fading out 'caa' so that the client will not relate the evocation of 'tuh' to 'caa'. Until when the child can now say, 'caa' - 'tuh' instead of 'cat'. Garry and Pear, suggested that it is better to teach the child large number of words that are not pronounced imperfectly than to spend time teaching a few perfect words. The child will perfect the pronunciation in course of his communication.

\section{Chaining}

Chaining is a stimulus-response-chain that connects one to another denoted by (S-R-S-RS-R-S-R-S) linking that holds firmly or together, (Martin and Pear, n.d.). It is a planned series of related responses that provides the cue for the subsequent responses that will earn reinforcement. It is either 'Backward' or Forward' exercise with rewarding of successive elements of response. This method can be used in training the retarded client (children) in dressing, grooming and verbal behavior. The activities are broken in to simpler and achievable objectives chained sequentially. There is the need for the counsellor to perform "task analysis: i.e. breaking down the chain into small manageable steps, (Martin and Pear, n.d.). For instance; when a student is to be taught how to pronounce the word 'milk'. The client will first pronounce ' $m$ ', then 'mi', then, 'mil', then, 'milk'. This is using forward chaining. In Backward chaining method, the client first pronounces, ' $\mathrm{k}$ ', then, ' $\mathrm{lk}$ ', then, 'ilk', then, 'milk'. According to Wikipedia the free Encyclopedia, chaining is an instructional procedure used in behavioural Psychology, Experimental analysis of behavior and applied behavioural analysis. It involves reinforcing individual's responses occurring in a sequence to form a complete behavior. There are two types of chaining. The homogeneous chaining when the responses are similar in each component, while heterogeneous chaining is where the responses differ in each link, (Cheney and Pierce, 2008).

\section{Extinction}

Ramalingam, (2006), defined extinction as the reduction and eventual disappearance of a learned or conditioned response after it is no longer paired with the unconditioned stimulus-response chain. Extinction is used to decrease the occurrence of behavior or response in a client. This technique is good in treating behaviours in children like, tantrums, crying, whining, and the face slapping. Because such behaviours are exhibited to attract the attention of others for whatever reason. Whenever the clients exhibit such behavior, they draw the attention of others around and the attention is a positive reinforcement to them. And whenever they exhibit such behavior, and are ignored, it 
becomes positive punishment and the repetition of the behavior will lessen. And whenever they are quite after exhibiting the behavior they should be reinforced for quietness. Such behaviours will gradually decrease and they should be encouraged to speak out their problems for the development of verbal skills and assertive behaviour. Martin and Pear, (2006), states that 'if the environment ignores the behaviour, then that behaviour will go away'. They emphasized that, extinction is most effective when paired with positive reinforcement for some desirable alternative behaviour. The principles of extinction is that, when a previous behavoiur is emitted and it is not followed by usual reinforcement that behavoiur tend to be lessen and it is likely to stop.

\section{Challenges affecting the use of Counselling Strategies against Learning Deficit}

Challenges of counselling strategies as behaviour modification cannot be over emphasized. Couple with in adequate trained professional counsellors, the application of such strategies will be nearly zero percent. The strategies are not familiar to most paracounsellors. It should be noted that, counsellors has limited time for clients, at list an hour or less depending on the maturity of the client and problem at hand. Students spend more time with parents, teachers and nannies. These categories of persons are not trained and cannot use these strategies to help their children and students. Where a counsellor helped a child using a strategy in a counselling center and the child goes home or class, the child cannot be assisted by teachers, parents and nannies since they are not trained and this will lead to extinction of learned activity.

Among other challenges is the application of other behavioural modifications procedures which sometimes results in accidental undesirable chaining of other behavior. For instance, where the counsellor/psychologist is trying to reduce excessive eating using chaining develops the habit of going to make phone calls while eating snacks to replace or supplement the food value with phone calls, (Martin and Pear, n.d.). Misuse of the counselling strategies by the inexperienced counsellors will result in developing another behavior. Onwuasoanya, (2006), observed that, there are some problems of inpatients from the unprofessional or inexperienced counsellors using some of strategies. Lack of displaying proper skills and inappropriate use of reinforcement on part of the counsellor hinders successful application of the strategies in clients.

\section{Conclusion}

In conclusion, whatever technique the counsellor uses for development of appropriate behaviour in a client, there are basic steps to follow. These include;

i. selection of the desired behavoiur to be increased or decreased,

ii. apply principles of the technique chosen sequentially,

iii. allow the desired behavoiur to be repeated before proceeding to next level of the experiment.

\section{Recommendations}

1. In adequate trained professional counselors with the knowledge and skills to handle children with learning deficit, 
2. Teachers, Namnies and Parents need to conversant with the application of other behavioural modifications procedures to help their child that exhibits such behavior,

3. Para-Counsellors should be sensitized on the application of the strategies. 


\section{Reference}

Abmbron, S. R. \& Salk, N. J. (1987). Child Development. (4 $4^{\text {th }}$ ed.) CBS College Publishers. New York

Board of Trustees, (2007). Learning Disability. University of Illinois.| 610 E John St | 217 $333 \quad 3704 \quad$ retrieved 26/12/2012 from: http://en.wikipedia.org/wiki/learning diability\#mw-head

Boerce, G. G. (1998). B. F. Skinner, Operant Conditioning. Retrieved: 9/1/2013. From: http://www.ship.edu/\%ccgoboeme

Chenery, C. \& Pierce, W. D. (2008). Behavior analysis and Learning. (4 ${ }^{\text {th }}$ ed.) Psychology Press. Wikipedia

Hartney, E. (2009). Health Diseases and Condition Content. Reviewed by Medical Review Board addiction.about.com

Martin, G. \& Pear, J. (2006). Behaviour Modification: What it is and how to do it. (newedt.), www.amazon.com/gp/aw/d/B004H Retrieved 2/1/2013.

Martin, L. G. \& Pear, J. J. (n.d.). Behaviour Modification: What it is and how to do it. $\left(8^{\text {th }}\right.$ edt. $)$, University of Manitoba

Learning Disability, (n.d.). "http://en.wikipedia.org/w/index.php?title=Learning disability\&oldid=528897130" Retrieved on $20^{\text {th }}$ Dec. 2012

Learning Disability, (n.d.). Learning Disability: Classification' and External Resources. http://en.m.wikipeadia.org/wiki/learning

Microsoft Encarta, (2009). 1993-2008 Microsoft Corporation. All rights reserved.

Morgan, C. T.; King, R. A.; Weisz, J. R. \& Schopler, J. (2006). Introduction to Psychology, ( $7^{\text {th }}$ edt.). New Delhi, Tata McGraw Hill Pub. Comp. Ltd

Onwuasoanya, P. N. (2006). Behaviour Modification Techniques in Counselling. Nsukka, Great AP Express Pub. Ltd.

Oxford Advance Learner's Dictionary, (2010). International Student's Edition, $\left(8^{\text {th }}\right.$ ed.) Oxford University Press.

Pressinger, R. W. (1997). Environmental Circumstances that can Damage the Development Brain. Journal of Pediatrics, 92 (1): 64-69. University of South Florida. Tampa, Florida.

Ramalingam, P. (2006). Academic Dictionary of Psychology. New Delhi, Academic Publishers. 
Shama, M. (2009). Short Attention Span. Simple Steps to Increase Child's Attention Span. www.articlesbase/atten

Uwe, E. A. (2005). Theories of Counselling and Psychotherapy. Callabar, EL-Sapphire Ltd. 
Shama, M. (2009). Short Attention Span. Simple Steps to Increase Child's Attention Span. www.articlesbase/atten

Uwe, E. A. (2005). Theories of Counselling and Psychotherapy. Callabar, EL-Sapphire Ltd. 